\title{
Elementos de un Plan de Estudios
}

\author{
Elements of a Study Plan
}

\author{
Noemi Vega-Lugo ${ }^{a}$, José Sergio Rodríguez-Martínez ${ }^{a}$, Ivette Flores-Jiménez ${ }^{b}$, Ruth Flores- \\ Jiménez ${ }^{b}$, Brenda Hurtado-Vega ${ }^{a}$
}

\begin{abstract}
:
By curriculum it is understood as «The set of objectives, pedagogical methods, contents and evaluation criterion that must regulate the teaching practice and its complements».

Its components are: academic program, which we can define as the ordered structure of the processes and procedures that form the curriculum; plans and programs of study, these allow to have an integral vision of the units and sub divisions of the content that should be treated in each one of them; design of plans and study programs within the context of the educational program, these being elaborated with methodological bases, containing the points described in advance and whose structure depends on the program and educational degree; rules of application of the study plans, which are conditioned to the regulations that prevail in the educational institution where the educational program (s) will be implemented; administrative rules of the study plans, like the previous point these will depend on what was agreed within the educational institution; to finish is the basic structure of the baccalaureate, undergraduate and / or postgraduate study plans where governed by the educational model that is managed.
\end{abstract}

\section{Keywords:}

Curriculum, Program, Structure

\section{Resumen:}

Por currículo se entiende como «El conjunto de objetivos, métodos pedagógicos, contenidos y criterios de evaluación que ha de regular la práctica docente y sus complementos».

Sus componentes son: programa académico, el cual podemos definir como la estructura ordenada de los procesos y procedimientos que forman el currículo; planes y programas de estudio, estos permiten tener una visión integral de las unidades y sub divisiones del contenido que se deberá tratar en cada uno de ellos; diseño de planes y programas de estudio dentro del contexto del programa educativo, siendo estos elaborados con bases metodológicas, conteniendo los puntos descritos con antelación y cuya estructura depende del programa y grado educativo; reglas de aplicación de los planes de estudio, mismas que están condicionadas a la normatividad que impera en la institución educativa donde se implementaran el o los programas educativos; reglas administrativas de los planes de estudio, al igual que el punto anterior estas dependerán de lo acordado dentro de la institución educativa; para finalizar se encuentra la estructura básica de los planes de estudio de bachillerato, licenciatura y/o posgrado donde regida por el modelo educativo que se maneje.

\section{Palabras Clave:}

Curriculo, Programa, Estructura

\footnotetext{
a Autor de Correspondencia, Universidad Autónoma del Estado de Hidalgo, Instituto de Ciencias Económico Administrativas, Email: Noemi Vega-Lugo noemivl@uaeh.edu.mx José Rodríguez sergior@uaeh.edu.mx Brenda Hurtado brenda_sol1212@hotmail.com ${ }^{\mathrm{b}}$ Universidad Autónoma del Estado de Hidalgo, Escuela Superior de Tlahuelilpan, Profesores Investigadores Ivette Flores Jiménez: ivette_flores7@hotmail.com; Ruth Flores Jiménez: ruthfj_22@hotmail.com
} 


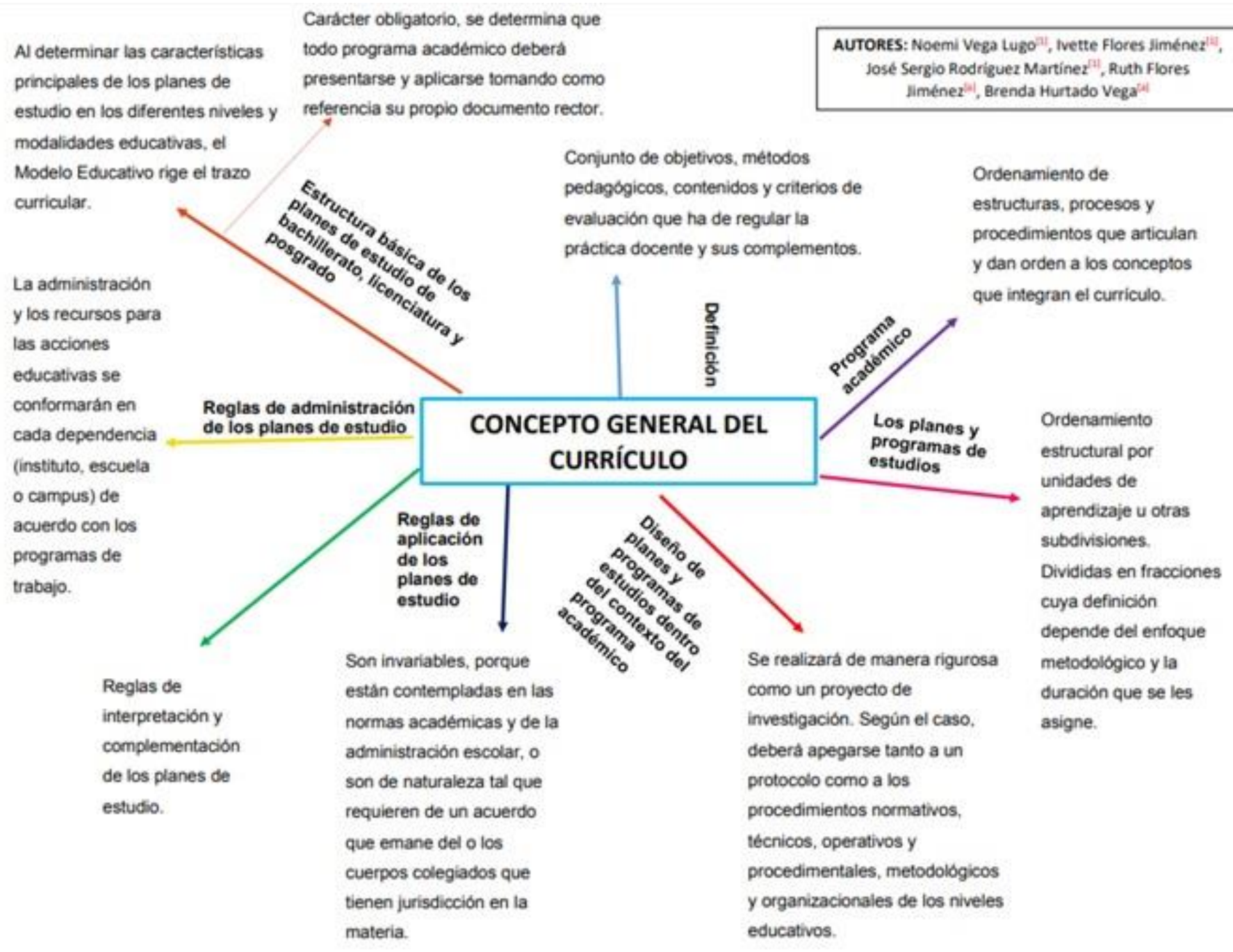

\section{Referencias}

[1] Hidalgo, U. A. (2015). Modelo Educativo. Pachuca, Hgo. : Universidad Autónoma del Estado de Hidalgo.

[2] Cruz Coria, E., Briones Juárez, A., Terrazas Juárez, A., \& González Espinoza, C. (2015). Programa Educativo de Licenciatura en Turismo.

México: UAEH. 